\title{
Mechanical Response of Metallic Glasses: Insights from In-situ High Energy X-ray Diffraction
}

\author{
Mihai Stoica, Jayanta Das, Jozef Bednarčik, Gang Wang, Gavin Vaughan, \\ Wei Hua Wang, Jürgen Eckert
}

The term "metallic glass" usually refers to a metallic alloy rapidly quenched in order to "freeze" its structure from the liquid state. A metallic glass is a metastable alloy, which lacks the symmetry typical for crystalline materials and at room temperature shows an amorphous liquid-like structure. Bulk metallic glasses (BMGs) represent a class of amorphous alloys. The most notable property of BMGs is their ultrahigh (near theoretical) strength and hardness. Because the known BMGs usually miss tensile plasticity and thus exhibit catastrophic failure upon tension it is important to understand deformation mechanisms involved and thus improve their performance. This aricle analyzes the use of synchrotron radiation for evaluating the elastic-plastic response of such materials.

\section{INTRODUCTION}

Due to the lack of crystalline structure, bulk metallic glasses (BMGs) may achieve interesting properties, including high strength and high hardness, excellent corrosion resistance, high wear resistance, very good soft magnetic properties, and, depending on composition, biocompatibility. ${ }^{1,2}$ The high strength of BMGs is sometimes accompanied by plastic deformation and their deformation and fracture mechanisms are quite different from crystalline materials. ${ }^{3-7}$ Bulk metallic glasses have strengths approaching the theoretical limit, ${ }^{8}$ but their plasticity at room temperature is typically very low. In uniaxial tension, the plastic strain is almost zero. ${ }^{9}$ For most of the known BMGs, plastic strain at room temperature is limited, less than $2 \%$, even under compression, resulting from pronounced shear localization and work softening. The lack of plasticity makes BMGs prone to catastrophic failure in load-bearing conditions and restricts their application. This also hinders the precise study of some fundamental issues in glasses, such as the deformation mechanism and the dynamics of plastic deformation, in which large plasticity is needed for detailed analysis.. ${ }^{9}$ Plastic deformation of metallic glasses at room temperature occurs through the formation and evolution of shear bands and is localized in thin shear bands. ${ }^{10}$ Therefore, brittleness is regarded as an intrinsic defect of metallic glasses.

Many methods have been developed and employed to rule out the de-

\section{How would you...}

...describe the overall significance of this paper?

$\infty$ This article analyzes the use of the synchrotron radiation for evaluating the elastic-plastic response of bulk metallic glasses (BMGs). BMGs are a new class of materials and their properties make them very attractive for applications. Here we show some

results obtained upon in-situ $x$-ray diffraction using synchrotron beam.

E...describe this work to a materials science and engineering professional with no experience in your technical specialty?

Due to the absence of a crystalline network, the BMGs may achieve high strength and elasticity, together with good wear and corrosion resistance. It is of great importance to understand deformation mechanisms involved and thus to improve their performance. Time resolved in-situ $x$ ray diffraction experiments may give crucial insights about the mechanical behavior up to atomic level.

3 ...describe this work to a layperson?

Bulk metallic glasses, novel materials with amorphous structure, have outstanding mechanical properties. The emergence of such properties can be studied by analyzing the $x$ ray diffraction patterns upon in-situ experiments. formation mechanisms characteristic to BMGs. ${ }^{11}$ Recently, characterization of amorphous materials by diffraction methods for the purpose of strain scanning was established. ${ }^{12}$ Several glasses were investigated since then, using different synchrotron sources: HASYLAB at Deutsches Elektronen-Synchrotron (DESY) Hamburg, Germany; European Synchrotron Radiation Facilities (ESRF) Grenoble, France; or Advanced Photon Source (APS) at Argonne National Laboratory, USA. Monochromatic hard x-rays with energies at $80-100$ $\mathrm{keV}$ were used for these experiments.

Ex-situ compression tests are usually performed to study the mechanical behavior of BMGs. This method is relatively simple and suitable for small samples. Tensile tests have technical limitations. First, for such tests a dog-bone shaped plate or rod sample is necessary, with a length of a few centimeters. This requires a BMG sample with quite large geometrical dimensions, which cannot be achieved by a poor glass former. The sample should be homogeneous, but in practice some small voids (as pores or oxides inclusions) may be present upon casting. Another limitation comes from the device used for tests-it is quite difficult to create a proper clamping system. Due to the difference in hardness between BMGs and the hardened steel used for tools, the BMG sample tends to slide from the grips.

See the sidebar for experimental details.

\section{DATA TREATMENT AND THEORETICAL BACKGROUND}

The elastic scattering intensity $I(Q)$ is measured as a function of the scattering vector (or wave vector) $Q$, which is defined as $4 \pi \sin \theta / \lambda$, where $\theta$ is half of 
the scattering angle (see Figure B) and $\lambda$ the wave length of the radiation. The structure factor can be written as:

$$
S(Q)=\frac{I(Q)}{N\langle f(Q)\rangle^{2}}
$$

where $N$ is the number of atoms, $f(Q)$ is the atomic scattering factor for $\mathrm{X}$-rays, and the angular brackets indicate averaging over the composition of the material. ${ }^{18}$ The real-space structural information available from $S(Q)$ is the pair distribution function $g(r)$, (PDF), in which $r$ is the distance from an average atom located at the origin. Without entering too much in details - the entire mathematic background can be found in several other works ${ }^{12-18}$ - one should mention that the pair distribution function is related to $S(Q)$ by a Fourier transform. It is also common to write the realspace structural information in terms of the radial distribution function (RDF), which is defined as $4 \pi r^{2} g(r)$. With this definition, the coordination number of a particular atomic shell of interest can be obtained by integrating the RDF over a suitably chosen range of $r$.

When an amorphous material is subjected to forces that create a macroscopic stress, both $S(Q)$ and $g(r)$ will be affected. For uniaxial loading, the changes in real space can be easy to anticipate. The tensile stress will tend to move atoms apart in the loading direction, and thus a peak in $g(r)$ for that direction will move to larger values of $r$. For a compressive stress, the opposite should happen. In the reciprocal space, $Q$, it is expected to shift toward lower values in the case of tensile stress and higher values when the sample is compressed. By analogy with the simple definition of engineering strain, the tensile strain for an applied stress $\sigma$ can be defined as:

$$
\varepsilon_{i}\left(\phi_{i}, \sigma\right)=\frac{Q\left(\varphi_{i}, 0\right)-Q\left(\varphi_{i}, \sigma\right)}{Q\left(\varphi_{i}, \sigma\right)}
$$

which is angular dependent. In the transverse direction, one expects a strain of the opposite sign due to the Poisson effect. A typical diffraction image of an amorphous sample is illustrated in Figure 1a. The amorphicity is proved by the absence of any clear ring. Then the image is integrated upon the polar coordinates $(s, \varphi)$. The integration is done by dividing the entire circle into $36 \mathrm{sec}-$ tions of $10^{\circ}$ each. The data were inte- grated with the help of the FIT2D software $^{19}$ and after integration the intensity curves were corrected for background, polarization, and inelastic Compton scattering. A typical diffraction pattern after integrating the diffraction image is presented in Figure 1b. The diffracted intensity $I$ as a function of wave vector $Q$ can then be transformed into the structure factor $S(Q)$. The index $i$ in Equation 2 takes the discrete values from 1 to 18 (due to the 36 section used for integration). Once the tensile load is applied, the round concentric halos from Figure

\section{EXPERIMENTAL SET-UP AND MEASURED BMGS}

The requirements for in-situ tensile tests under synchrotron radiation are: proper sample, proper testing device, access at the hard x-ray source, and proper geometric set-up. In our case, the samples used for testing were cast as amorphous plates which further were machined by the spark erosion method in order to obtain a dog-bone shaped specimen with 10 $\mathrm{mm} \times 2 \times 1 \mathrm{~mm}^{2}$ reduced section (Figure A). The dog-bone shaped specimen was strained using a tensile module from Kammrath and Weiss $\mathrm{GmbH}$, which can achieve a maximum load of $5 \mathrm{kN}$. The room temperature in-situ X-ray diffraction experiments were performed on the wiggler beamline BW5 at the DORIS positron storage ring (HASYLAB at DESY, Hamburg, Germany) using monochromatic synchrotron radiation of $103.8 \mathrm{keV}(\lambda=0.0119$ $\mathrm{nm})$. The layout of the experimental setup is shown in Figure B. The measured samples were exposed for $10 \mathrm{~s}$ to the well collimated incident beam having a cross section of $1 \times 1$ $\mathrm{mm}^{2}$. Two dimensional (2-D) $\left(2,300 \times 2,300\right.$ pixels, $150 \times 150 \mu \mathrm{m}^{2}$ pixel size $) \mathrm{x}$-ray diffraction (XRD) patterns were collected using a MAR 345 2-D image plate detector carefully mounted orthogonally to the $\mathrm{X}$-ray beam. The diffraction pattern from $\mathrm{LaB}_{6}$ was used to calibrate the sample-to-detector distance $D$ and tilting of the image plate detector with respect to the beam axis.

The BMGs studied using this set-up were of the composition $\mathrm{Zr}_{64.13} \mathrm{Cu}_{15.75} \mathrm{Ni}_{10.12} \mathrm{Al}_{10}{ }^{13,14}$ At the same location, BW5 beamline, and with the same geometrical set-up, Wang et al. ${ }^{15}$ measured $\mathrm{Zr}_{62} \mathrm{Al}_{8} \mathrm{Ni}_{13} \mathrm{Cu}_{17}$ and $\mathrm{La}_{62} \mathrm{Al}_{14}\left(\mathrm{Cu}_{5 / 6} \mathrm{Ag}_{1 / 6}\right)_{14} \mathrm{Co}_{5} \mathrm{Ni}_{5}$ BMGs using x-ray radiation with $\lambda=0.012389 \mathrm{~nm}$. In-situ tensile tests at BW5 were also reported by Mattern et al. ${ }^{16}$ Their samples were amorphous ribbons with the compositions $\mathrm{Cu}_{50} \mathrm{Zr}_{50}$ and $\mathrm{Cu}_{65} \mathrm{Zr}_{35}$ and the radiation had $\lambda=0.01265 \mathrm{~nm}$. Prior to these experiments, few others were performed in compression. At ESRF Grenoble, Poulsen et al. ${ }^{12}$ investigated the $\mathrm{Mg}_{60} \mathrm{Cu}_{30} \mathrm{Y}_{10}$ BMGs and Das et al. ${ }^{17} \mathrm{Cu}_{47.5} \mathrm{Zr}_{47.5} \mathrm{Al}_{5}$ and $\mathrm{Zr}_{55} \mathrm{Cu}_{20} \mathrm{Ni}_{10} \mathrm{Al}_{10} \mathrm{Ti}_{5}$ BMGs. The wave length of the radiation used for these experiments was $\lambda=0.01412 \mathrm{~nm}$ for $\mathrm{Mg}$-based glasses and $\lambda=0.0155$ $\mathrm{nm}$ for $\mathrm{Zr}$-based glasses. Other BMGs, $\mathrm{Zr}_{57} \mathrm{Ti}_{5} \mathrm{Cu}_{20} \mathrm{Ni}_{8} \mathrm{Al}_{10}$, were tested in uniaxial compressive load using a $0.0154 \mathrm{~nm}$ radiation at Advanced Photon Source at Argonne National Laboratory by Hufnagel, Ott, and Almer. ${ }^{18}$ For further comparisons between measured data it is important to point out that for almost all mentioned experiments, the diffraction

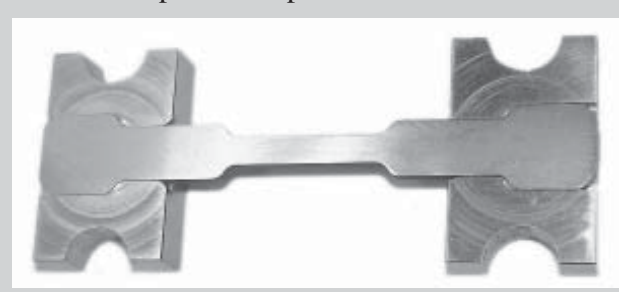

Figure A. A dog-bone shaped sample used for tensile tests.

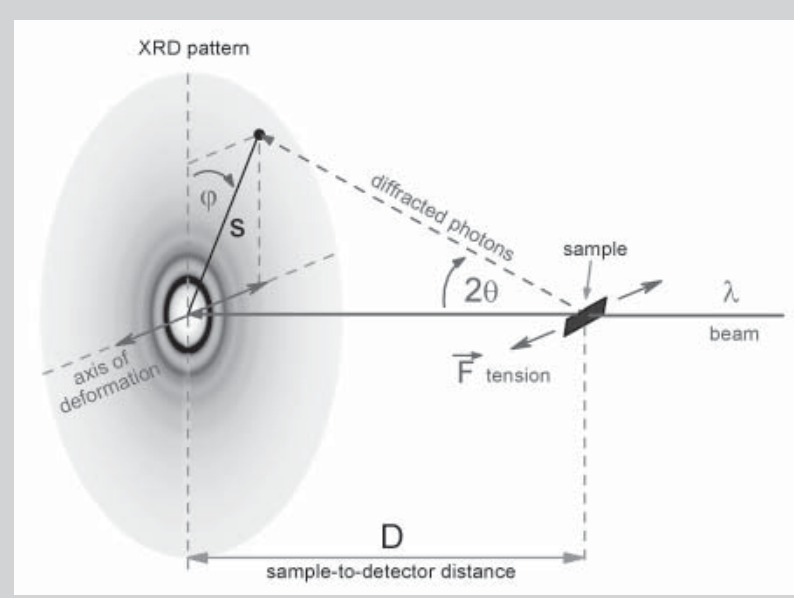
images were recorded with the same kind of 2-D detector, the MAR 345 image plate. The only exception came from Das et al. ${ }^{17}$ They used as detector a fast response low noise charge coupled device (FReLoN CCD). However, the primary diffraction images were integrated with the help of the FIT2D software. ${ }^{19}$

Figure B. A sketch of a typical in-situ tensile experiment. 


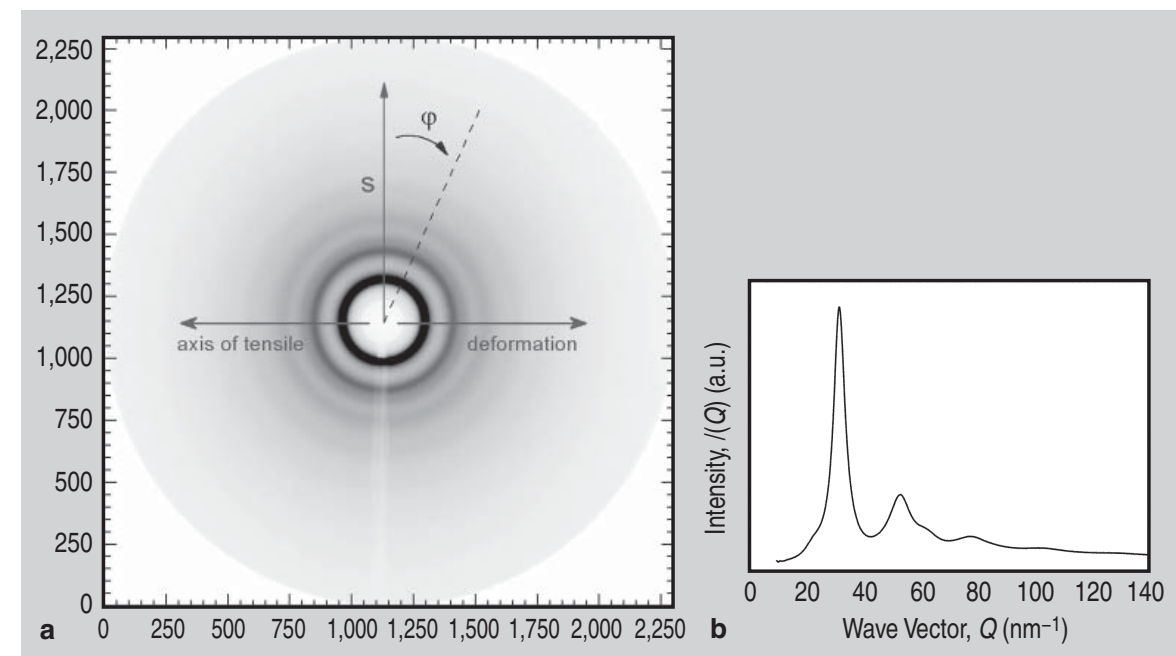

Figure 1. (a) Diffraction image as recorded by the MAR 345 2D image plate detector. The polar coordinates $(s, \varphi)$ and the axis of tensile deformation are depicted. (b) X-ray diffraction pattern resulting from integration among the polar coordinates $(s, \varphi)$ of the image presented in (a). Both (a) and (b) are typical for metallic glasses and here they were recorded for $\mathrm{Zr}_{64.13} \mathrm{Cu}_{15.75} \mathrm{Ni}_{10.12} \mathrm{Al}_{10} \mathrm{BMG}$.

1a become elliptical and the asymmetry is higher as the load increases. Then the angular variation of the strain can be fitted with: ${ }^{13}$

$$
\begin{gathered}
\varepsilon(\varphi, \sigma)=\varepsilon_{11} \sin ^{2} \varphi \\
+\varepsilon_{12} \sin \varphi \cos \varphi+\varepsilon_{22} \cos ^{2} \varphi
\end{gathered}
$$

As a result, the strain tensor can be determined, and the axial $\varepsilon_{11}$, tangential $\varepsilon_{22}$, and in-plane shear component $\varepsilon_{12}$ can be derived. Taking into account the set-up presented in Figure B, $\varphi=90^{\circ}$ corresponds to the axial stress and $\varphi=$ $0^{\circ}$ to the tangential stress. Components not in the plane perpendicular to the incoming beam can be determined by rotating the specimen around an axis perpendicular to the incoming beam. In the more general case of a multiaxial stress state, the complete strain tensor can be determined from measurement of the strain in various directions. ${ }^{14,18}$

The same analysis can be done in the real space. However, experimentally a good correlation was observed between the two sets of data. Dmowski and Ega$\mathrm{mi}^{20}$ pointed out that in the presence of structural anisotropy, it is necessary to expand the PDF into spherical harmonics, otherwise systematic errors may occur especially in the first neighborhood.

\section{STRAIN ANALYSIS}

\section{Tensile Tests and Reciprocal Space}

The 2-D diffraction pattern of as-cast $\mathrm{Zr}_{64.13} \mathrm{Cu}_{15.75} \mathrm{Ni}_{10.12} \mathrm{Al}_{10}$ exhibits the diffuse scattering pattern typical for me- tallic glasses and confirms the presence of glassy structure without any hint of crystalline inclusions (see Figure 1). The symmetric circular diffraction pattern is characteristic for the samples prior to applying tensile stress. With increasing tensile load it becomes elliptical. The changes are most pronounced for the first and strongest diffuse ring (halo) appearing in the 2-D XRD pattern. To describe such changes more quantitatively one has to construct the set of symmetrized intensity distributions as described previously and trace the change in the first peak position as a function of azimuth angle, $\varphi$, and tensile stress, $\sigma$. It should be noted here that no changes were observed between seven diffraction patterns, independently acquired along the length of the sample (in $1 \mathrm{~mm}$ steps), when holding the load at a fixed value of external stress. The experimental scatter of the measured strain values at each different stress level for seven independent locations along the gauge length is shown as error bars in Figures 2 and 3. From Figure 2 it is evident that the asymmetry of the first diffuse maximum increases with increasing load. The decrease in the peak position with increasing tensile stress (the curve corresponding to $\varphi=90^{\circ} \mathrm{Fig}$ ure 1) reflects the fact that atoms move apart along the tensile direction. An opposite behavior is seen in transversal direction (the curve corresponding to $\varphi=0^{\circ}$ in Figure 1). Figure 2 shows the angular variation of the strain at a given stress $\sigma$ as calculated from the rel- ative change in the position of the first peak using Equation 2. The fit of the experimental data to Equation 3 yields two components of the strain tensor, $\varepsilon_{11}$ and $\varepsilon_{22}$ (the axial and tangential components, respectively). The stress-strain curves as observed for different strain tensor components are plotted in Figure 4. Within the experimental error all of them show a linear behavior, indicating the elastic regime of the tensile deformation for the investigated specimens. The samples fractured at a stress of about 1,500 MPa with no sign of yielding, despite the fact that the compressive yield strength of this BMG was reported to be $1,690-1,851 \mathrm{MPa},{ }^{21}$ indicating a significant strength asymmetry for this BMG. The maximum axial strain $\left(\varepsilon_{11}\right)$ is $1.50 \pm$ $0.01 \%$. The elastic modulus determined in tensile mode is $E_{11}=94 \pm 1 \mathrm{GPa}$ and the experimentally determined Poisson's ratio $v=-\varepsilon_{22} / \varepsilon_{11}$ is $0.325 \pm 0.01$.

Using the diffraction data by insitu high-energy XRD, the tensile modulus and Poisson's ratio can be accurately evaluated in the case of $\mathrm{Zr}_{62} \mathrm{Al}_{8} \mathrm{Ni}_{13} \mathrm{Cu}_{17}$ and $\mathrm{La}_{62} \mathrm{Al}_{14}\left(\mathrm{Cu}_{5 / 6}\right.$ $\left.\mathrm{Ag}_{1 / 6}\right)_{14} \mathrm{Co}_{5} \mathrm{Ni}_{5}$ BMGs. ${ }^{15}$ As in the case of $\mathrm{Zr}_{64.13} \mathrm{Cu}_{15.75} \mathrm{Ni}_{10.12} \mathrm{Al}_{10}$, no tensile plasticity was observed, despite the fact that both $\mathrm{Zr}$-glasses are rather deformable in compression. ${ }^{15,21}$ The strains determined from the diffraction data of tensile/transverse directions for the $\mathrm{Zr}_{62} \mathrm{Al}_{8} \mathrm{Ni}_{13} \mathrm{Cu}_{17}$ and $\mathrm{La}_{62} \mathrm{Al}_{14}\left(\mathrm{Cu}_{5 / 6} \mathrm{Ag}_{1 / 6}\right)_{14} \mathrm{Co}_{5} \mathrm{Ni}_{5}$ BMGs are presented in Figure 5. There one can see the good linear behavior and basically no sign of yielding. By linearly fitting the points and calculating the ratio of strains between the transverse and tensile directions for each alloy, the tensile elastic modulus and Poisson's ratio were obtained, about $83 \mathrm{GPa}$ and 0.37 for $\mathrm{Zr}_{62} \mathrm{Al}_{8} \mathrm{Ni}_{13} \mathrm{Cu}_{17} \mathrm{BMG}$ and $34 \mathrm{GPa}$ and 0.36 for $\mathrm{La}_{62} \mathrm{Al}_{14}\left(\mathrm{Cu}_{5 / 6} \mathrm{Ag}_{1 / 6}\right)_{14} \mathrm{Co}_{5} \mathrm{Ni}_{5}$ $\mathrm{BMG}$, respectively.

Using the same method, Mattern et al. ${ }^{16}$ measured upon tensile loading amorphous ribbons with the compositions $\mathrm{Cu}_{50} \mathrm{Zr}_{50}$ and $\mathrm{Cu}_{65} \mathrm{Zr}_{35}$. The corresponding data for $\mathrm{Cu}_{50} \mathrm{Zr}_{50}$ are presented in Figure 6. There one can see again a good linear behavior up to the highest value before fracture of the strain with applied stress and no sign of plastic deformation. The Young's modulus $E=$ $63 \mathrm{GPa}$ and the Poisson's ratio $v=0.31$ were calculated directly from the slopes 
of the axial and tangential components $\varepsilon_{11}$ and $\varepsilon_{22}$ vs. stress. The other glass, $\mathrm{Cu}_{65} \mathrm{Zr}_{35}$, behaves similarly.

\section{Compression Tests and Reciprocal Space}

The evaluation of the elastic tensor by synchrotron radian was established by Poulsen et al. ${ }^{12}$ At ESRF, they measured several $\mathrm{Mg}_{60} \mathrm{Cu}_{30} \mathrm{Y}_{10}$ BMGs under compressive load. A good linear dependence of the strain as a function of the applied stress was also found. ${ }^{12}$ This BMG was known to be brittle, ${ }^{22}$ and, as a consequence, the samples failed at the end of the elastic regime. Hufnagel, Ott, and Almer ${ }^{18}$ measured, at APS, $\mathrm{Zr}_{57} \mathrm{Ti}_{5} \mathrm{Cu}_{20} \mathrm{Ni}_{8} \mathrm{Al}_{10}$ BMGs loaded in uniaxial compression. They used the structure factor $S(Q)$ recorded for the loading direction from many $\mathrm{x}$-ray scattering patterns taken at various stresses during incremental loading from $0 \mathrm{MPa}$ to $1,080 \mathrm{MPa}$ (approximately $60 \%$ of the yield stress for this alloy ${ }^{18}$ ) and back to zero. As the compressive stress increases, the largest peak in $S(Q)$ shifts to larger $Q$ in the loading direction. The opposite trend was observed for $S(Q)$ in the transverse direction. As a result, the axial component $\varepsilon_{11}$ of the strain tensor became negative and the tangential component $\varepsilon_{22}$ became positive.

The results indicated that the strain increases linearly with increasing compressive stress. A straight-line fit to the data yields an elastic modulus of $E=87$ $\pm 2 \mathrm{GPa}$, in good agreement with values for $E$ determined by macroscopic measurements on closely related amorphous alloys. ${ }^{18}$ Data for the transverse direction are also in a linear dependence with the applied stress and from both directions one can obtain the value for Poisson's ratio of $v=0.34 \pm 0.01$, also in reasonable agreement with the macroscopic measurements. ${ }^{18}$

Strain scanning by $\mathrm{x}$-ray diffraction beyond the Hookean limit, in order to investigate the plastic yielding phenomena of two different BMGs at higher resolution, was done by Das et al. ${ }^{17}$ The alloys are "plastic" $\mathrm{Cu}_{47.5} \mathrm{Zr}_{47.5} \mathrm{Al}_{5}{ }^{23}$ and macroscopically "brittle" $\mathrm{Zr}_{55} \mathrm{Cu}_{20} \mathrm{Ni}_{10} \mathrm{Al}_{10} \mathrm{Ti}_{5}{ }^{24}$ BMGs. The experiments have been performed at ESRF Grenoble, France, and the detailed experimental setup is described elsewhere. ${ }^{17}$ The diffraction patterns showed the elliptical nature of the ring after loading compared to a circular feature of the unloaded state, indicating a decrease of the atomic spacing of the nearest neighbors along the loading axis. The data analysis has been performed by the $Q$-space method in reciprocal space, as described earlier. In the case of each integrated intensity $I(Q)$, the shift of the first halo was determined with respect to the unloaded condition.

Figure $7 \mathrm{a}$ and $\mathrm{b}$, taken from Reference 17, shows the evolution of the different atomic-scale strain components $\left(\varepsilon_{11}, \varepsilon_{22}\right.$ and $\left.\gamma_{12}=\varepsilon_{12}\right)$ with applied stress for $\mathrm{Zr}_{55} \mathrm{Cu}_{20} \mathrm{Ni}_{10} \mathrm{Al}_{10} \mathrm{Ti}_{5}$ and $\mathrm{Cu}_{47.5} \mathrm{Zr}_{47.5} \mathrm{Al}_{5}$, respectively. In the case of $\mathrm{Zr}_{55} \mathrm{Cu}_{20} \mathrm{Ni}_{10} \mathrm{Al}_{10} \mathrm{Ti}_{5}$, the strain components in the loading direction $\left(\varepsilon_{11}\right)$ and transverse direction $\left(\varepsilon_{22}\right)$ increase.
However, the shear component $\gamma_{12}$ values remain close to zero. The increment of $\varepsilon_{11}$ and $\varepsilon_{22}$ strains slightly deviates from linearity after 1,400 MPa (a dotted line has been drawn to show the linearity of the elastic stress-strain relationship), and the sample broke at 1,740 $\mathrm{MPa}$ with an axial strain $\varepsilon_{11}=-0.0174$ and a transverse strain $\varepsilon_{22}$ of +0.00675 . This strength value is similar to the macroscopic yielding (MY) of this alloy at 1,727 $\mathrm{MPa}$, as observed earlier. ${ }^{24}$ $\mathrm{Cu}_{47.5} \mathrm{Zr}_{47.5} \mathrm{Al}_{5}$ shows (Figure $7 \mathrm{~b}$ ) a very similar stress-strain relationship. However, the nonlinear stress-strain behavior starts at around 1,200 $\mathrm{MPa}$ and, finally, the elastic strain saturates at a stress of 1,506 MPa with an axial strain $\varepsilon_{11}=-0.0150$ and a transverse strain

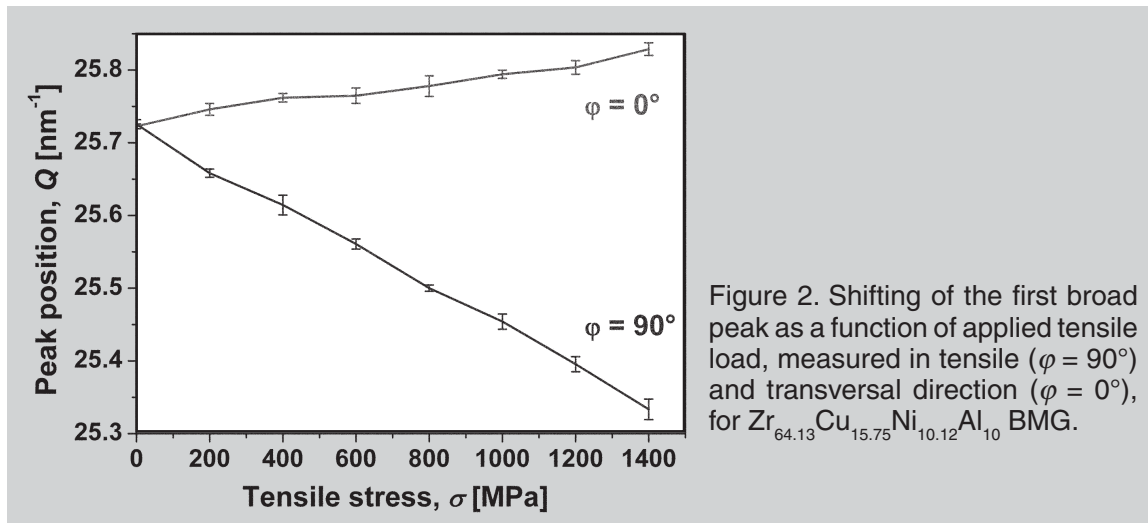

Figure 3. Angular dependence of the strain determined at various stages of tensile deformation of $\mathrm{Zr}_{64.13} \mathrm{Cu}_{15.75} \mathrm{Ni}_{10.12}$ $\mathrm{Al}_{10} \mathrm{BMG}$, as calculated from the relative change in the position of the first peak using Equation 2.
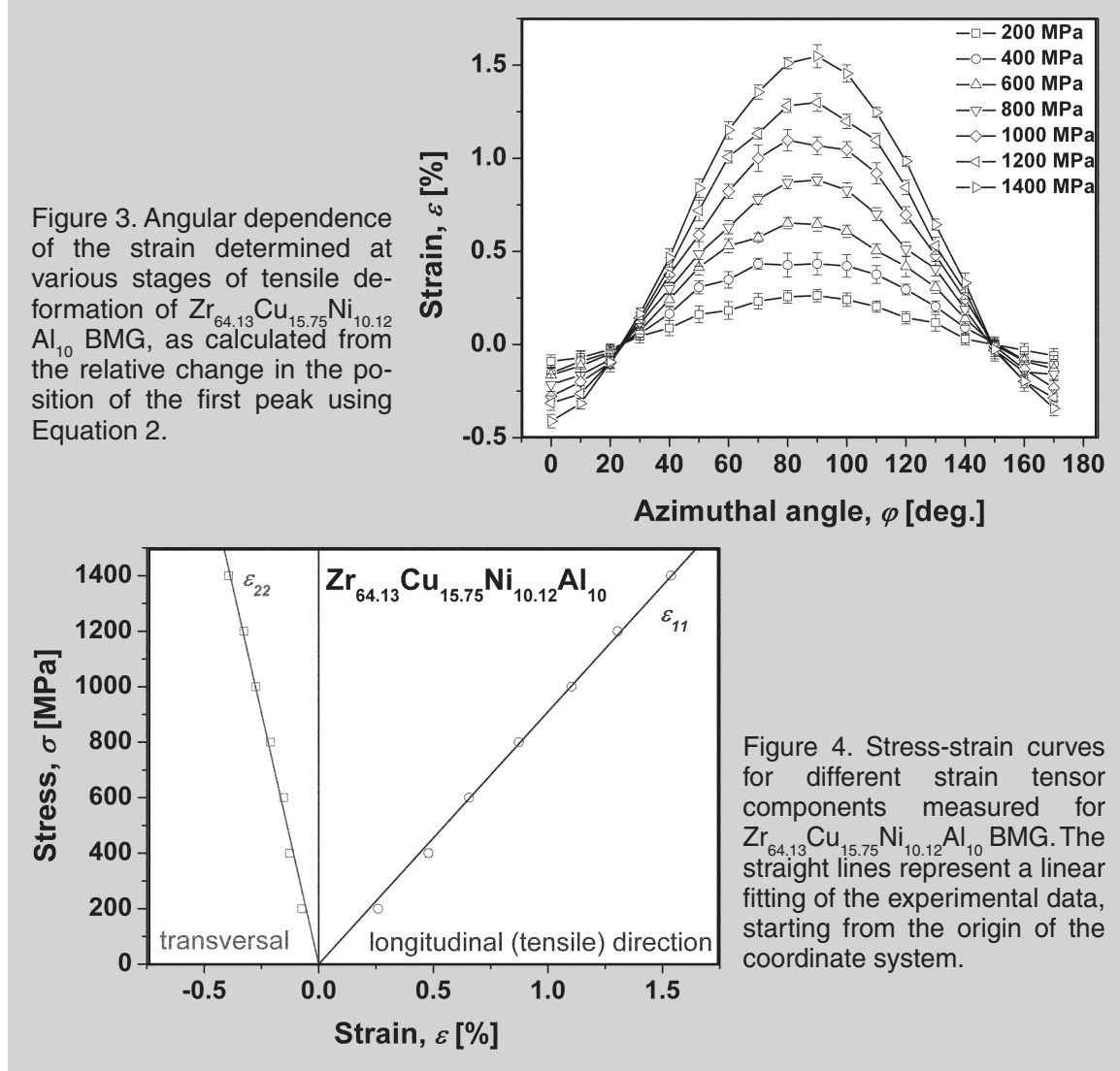
$\varepsilon_{22}=+0.0056$ without alteration of the shear component $\gamma_{12}$, which is close to 0 . The test was stopped at $1,700 \mathrm{MPa}$ at a plastic strain of about $0.6 \%-0.7 \%$. Note that the microscopic yield stress has been measured to be 1,547 $\mathrm{MPa}$ for $\mathrm{Cu}_{47.5} \mathrm{Zr}_{47.5} \mathrm{Al}_{5}$, as reported earlier. ${ }^{23}$ Therefore, the stress required for mac- roscopic yielding under compression and the saturation of the elastic strain at the atomic scale is consistent for both the investigated alloys.

\section{Strain Analysis from the Real Space}

The strain analysis can be done using

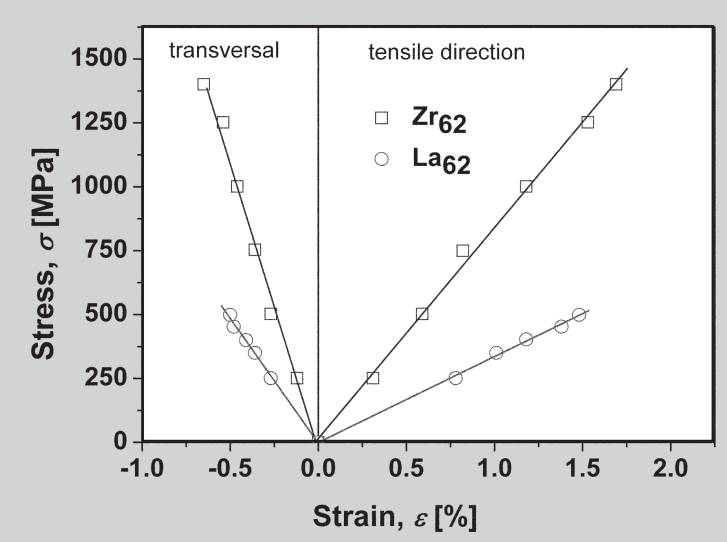

Figure 5. The strains determined from the diffraction data of tensile/transverse directions for $\mathrm{Zr}_{62} \mathrm{Al}_{8} \mathrm{Ni}_{13} \mathrm{Cu}_{17}$ and $\mathrm{La}_{62} \mathrm{Al}_{14}\left(\mathrm{Cu}_{5 / 6} \mathrm{Ag}_{1 / 6}\right){ }_{14} \mathrm{CO}_{5} \mathrm{Ni}_{5}$ BMGs. As for Figure 4, the straight lines represent the linear fitting of the experimental data.

Figure 6. Strain vs. applied stress of $\mathrm{Cu}_{50} \mathrm{Zr}_{50}$ glass measured by $x$-ray diffraction. Here also the linear fitting is presented by the straight lines.
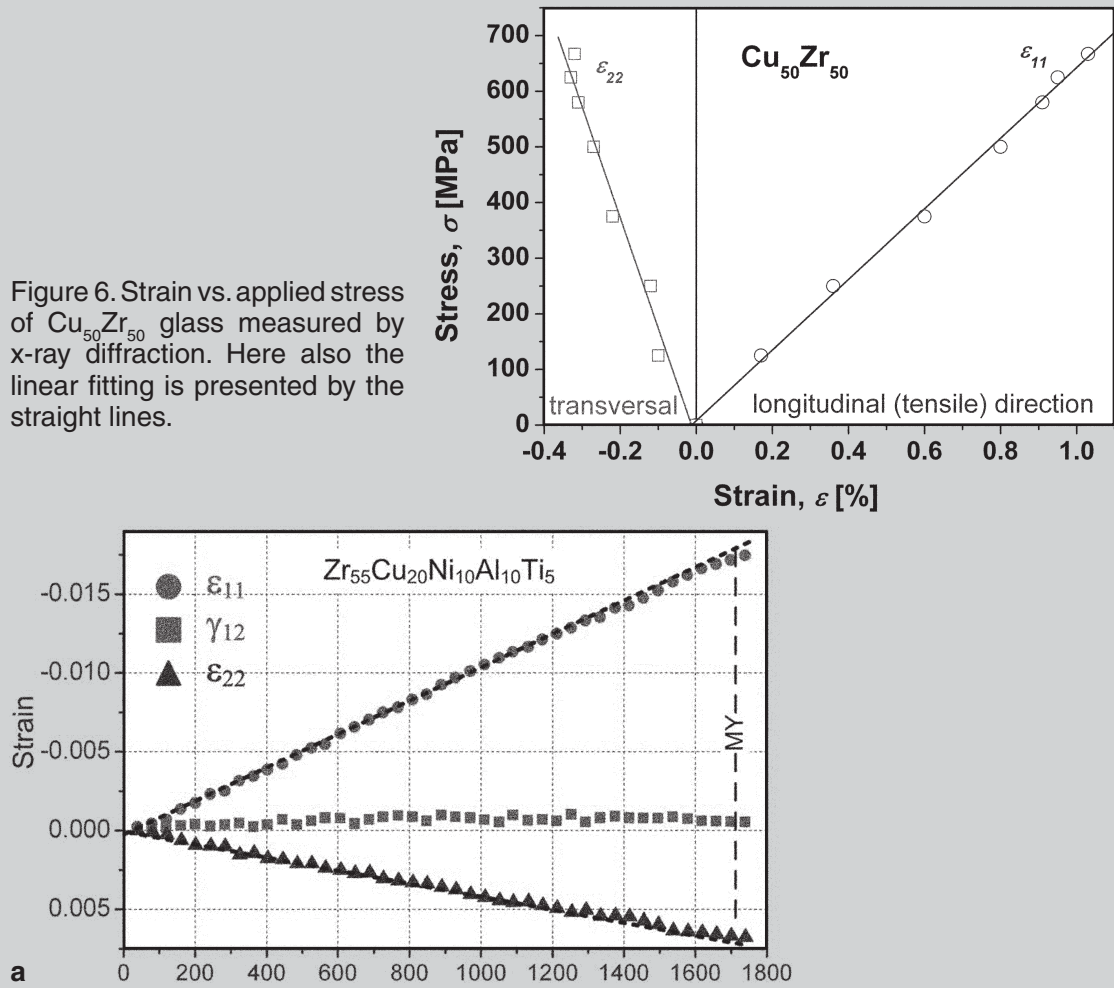

Figure 7. Evolution of elastic strain components of (a) $\mathrm{Zr}_{55} \mathrm{Cu}_{20} \mathrm{Ni}_{10} \mathrm{Al}_{10} \mathrm{Ti}_{5}$ and (b) $\mathrm{Cu}_{47.5} \mathrm{Zr}_{47.5} \mathrm{Al}_{5}$ during compressive loading. The increment of $\varepsilon_{11}$ and $\varepsilon_{22}$ strains deviates from linearity on atomic scale before the onset of macroscopic yielding (MY). The figure is taken from Reference 17.

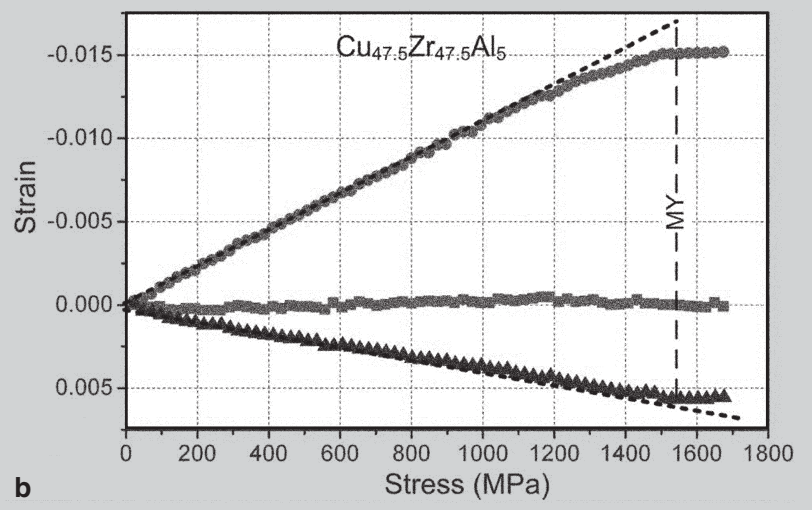

the data from the real space. For that, one has to calculate the structure factor $S(Q)$ or pair correlation function $g(r)$ as described earlier. An example is given in Figure 8, which represents $g(r)$ of $\mathrm{Zr}_{64.13} \mathrm{Cu}_{15.75} \mathrm{Ni}_{10.12} \mathrm{Al}_{10}$ BMG at different stages of deformation. The first peak in $g(r)$ (see the inset) shifts to larger $r$ with increasing load, as expected, and the transverse data (not shown) show the opposite trend. According to different authors, ${ }^{18,20}$ the peak positions in $g(r)$ are difficult to determine accurately because the peaks at low $r$ are asymmetric while those at larger $r$ are rather broad, which leads to significant scatter in the measured strain. A more robust technique proposed by Hufnagel, Ott, and Almer ${ }^{18}$ is to focus not on the tops of the peaks, but on the places where $g(r)=1$. These crossing points are less sensitive to the effects of asymmetry and can be accurately determined even for peaks at large $r$. Although no dependence of strain on $r$ was observed, it is interesting that the strain determined from the lowest value of $r$ at which $g(r)=1$ is consistently smaller in magnitude than the strains determined at larger values of $r{ }^{18}$ This is related to the asymmetry of the peaks, due by the changes in the interatomic bonding lengths. More, the asymmetry becomes more pronounced when the applied stress is increasing.To investigate this further, one has to move to RDF and try to deconvolute the first peak which corresponds to the first coordination shell. We cannot unambiguously identify the atomic pairs contributing to the first peak in the RDF, but we can make some reasonable approximations for this $\mathrm{Zr}_{64.13} \mathrm{Cu}_{15.75} \mathrm{Ni}_{10.12} \mathrm{Al}_{10}$ alloy. First, because the contribution of each atomic pair to the RDF is weighted by the atomic scattering factors of the elements and by their concentration, we can neglect the influence of $\mathrm{Al}$, because it has a low atomic number and is present at relatively low concentration. Second, the separation of the atoms in each pair is related to the sum of their atomic radii; since $\mathrm{Cu}$ and $\mathrm{Ni}$ are nearly the same size (1.28 and 1.25 $\AA$, respectively), ${ }^{25}$ their contributions are indistinguishable. It is almost clear that $\mathrm{Zr}-\mathrm{Zr}, \mathrm{Zr}-\mathrm{Cu}$, and $\mathrm{Zr}-\mathrm{Ni}$ are the dominant atomic pairs which constitute the first coordination shell of the PDFs, so only two partials $[\mathrm{Zr}-(\mathrm{Cu}, \mathrm{Ni})$ and 
$(\mathrm{Zr}-\mathrm{Zr})]$ need to be resolved. Figure 9a shows the result of the deconvolution for the first shell of undeformed $\mathrm{Zr}_{64.13} \mathrm{Cu}_{15.75} \mathrm{Ni1}_{0.12} \mathrm{Al}_{10}$. The center of the first peak was estimated to be at $2.68 \AA$ corresponding to $\mathrm{Zr}-(\mathrm{Cu}, \mathrm{Ni})$ atomic pairs. The major component centered at $3.14 \AA$ originates from $\mathrm{Zr}-\mathrm{Zr}$ atomic pairs. As can be seen from Figure 9b, an increase in tensile stress shifts both peaks toward higher $r$ values. This proves that tensile stress increases the average atomic distances along the loading direction. Similar results were obtained in the case of the other metallic glasses discussed here; in the case of compressive loadings, the average atomic distances along the loading direction became smaller. ${ }^{18}$

\section{DATA SUMMARY, COMPARISON, AND DISCUSSION}

All of the above discussion assumes that the amorphous material is isotropic. This is often the case for amorphous alloys, but counterexamples can be found in thin films and in bulk alloys subjected to processing that renders them anisotropic. Even in these cases the deviation from isotropy is usually small and the scattering data are analyzed using the isotropic assumption. For the data presented here it was assumed that one can neglect the anisotropy induced by the small uniaxial elastic strain. Hufnagel, Ott and Almer mention ${ }^{18}$ that a full treatment would involve the application of cylindrical distribution functions to properly handle the symmetry.

Table I summarizes the values of Young's modulus $E$ and Poisson's ratio $v$ for the metallic glasses analyzed here. Two types of data are presented: measured by XRD using synchrotron radiation and measured using ultrasound methods or macroscopic tensile/compression tests. Basically, there always are differences in the constants measured using the two methods.

For compressive loads, as mentioned also by Hufnagel, Ott, and Almer, ${ }^{18}$ Poulsen and co-workers ${ }^{12}$ demonstrated for the first time that the theoretical considerations used in this work are essentially correct, by measuring elastic strain and strain distributions in $\mathrm{Mg}_{60} \mathrm{Cu}_{30} \mathrm{Y}_{10}$ BMG. They showed that the strain measured in both reciprocal and real space
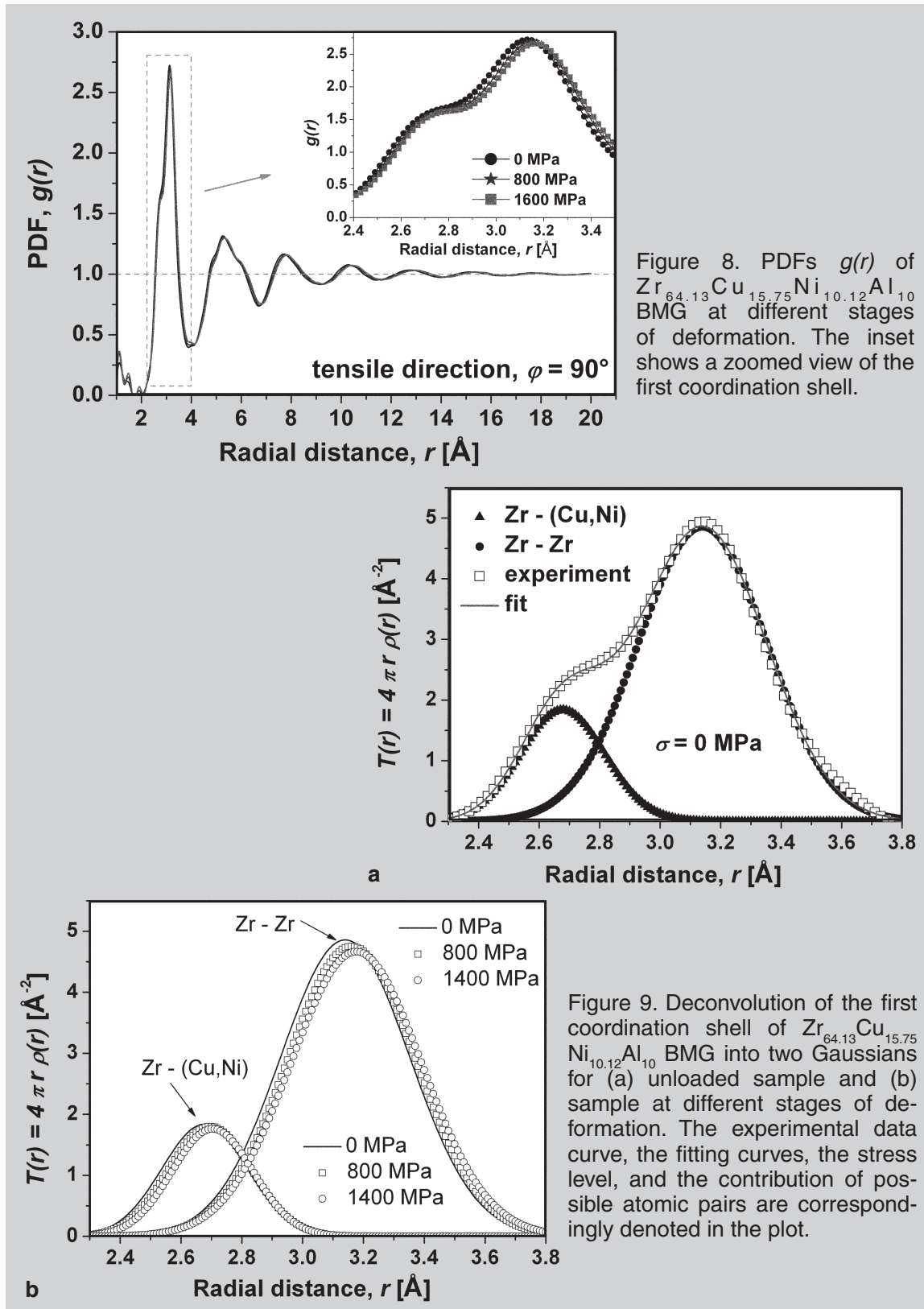

increased linearly with uniaxial stress. The strain measured from the position of the first peak in $I(Q)$ showed good agreement with strain calculated based on macroscopic measurements of Young's modulus E. However, the strain calculated from $g(r)$ showed a pronounced dependence on $r$, being the smaller for the first near neighbor peak and increasing with peaks at higher $r$ to asymptotically approach the strain calculated from the $I(Q)$ peak position. The strain calculated from the third $g(r)$ peak was even 2.7 times as large as that from the first peak. Poulsen et al. attributed this observation to unspecified "structural rearrangements on the length scale of 4-10 $\AA$. ."12 Hufnagel and co-workers ${ }^{18}$ observed as well that the strain in the nearest-neighbor shell is smaller than that at longer scales, the effect being smaller than that reported by Poulsen et al. ${ }^{12}$ They propose that "the difference between the stiffness of the nearest-neighbor atomic environment and that over longer length scales can be attributed to the effect of topological rearrangements in the nearest-neighbor environments of a relatively small fraction of the atoms, without the need to invoke significant structural rearrangements over longer length scales." 18

The differences in mechanical constants measured using different techniques are even higher when the samples were subjected to tensile loads (see Table I). Here the stiffness of the neighbor cells becomes more important. Basically, upon tensile tests no sign of plastic deformation was observed. In 
Table I. The Values for Young's Modulus $E$ and Poisson's Ratio $v$ for the Metallic Glasses Presented in this Paper

\begin{tabular}{|c|c|c|c|}
\hline Composition & Experiment & $E(\mathrm{GPa})$ & $v$ \\
\hline $\mathrm{Zr}_{64.13} \mathrm{Cu}_{15.75} \mathrm{Ni}_{10.12} \mathrm{Al}_{10}$ & $\begin{array}{c}\text { Tensile, synchrotron }{ }^{13} \\
\text { Ultrasound }^{13}\end{array}$ & $\begin{array}{l}94 \\
78\end{array}$ & $\begin{array}{l}0.325 \\
0.377\end{array}$ \\
\hline $\mathrm{Zr}_{62} \mathrm{Al}_{8} \mathrm{Ni}_{13} \mathrm{Cu}_{17}$ & $\begin{array}{l}\text { Tensile, synchrotron } \\
\text { Ultrasound }^{15}\end{array}$ & $\begin{array}{l}83 \\
80\end{array}$ & $\begin{array}{l}0.37 \\
0.38\end{array}$ \\
\hline $\mathrm{La}_{62} \mathrm{Al}_{14}\left(\mathrm{Cu}_{5 / 6} \mathrm{Ag}_{1 / 6}\right)_{14} \mathrm{Co}_{5} \mathrm{Ni}_{5}$ & $\begin{array}{l}\text { Tensile, synchrotron } \\
\text { Ultrasound } \\
{ }^{15}\end{array}$ & $\begin{array}{l}34 \\
35\end{array}$ & $\begin{array}{l}0.36 \\
0.36\end{array}$ \\
\hline $\mathrm{Cu}_{50} \mathrm{Zr}_{50}$ & $\begin{array}{l}\text { Tensile, synchrotron }{ }^{16} \\
\text { Ultrasound }\end{array}$ & $\begin{array}{l}63 \\
83\end{array}$ & $\begin{array}{l}0.31 \\
0.384\end{array}$ \\
\hline $\mathrm{Cu}_{65} \mathrm{Zr}_{35}$ & $\begin{array}{c}\text { Tensile, synchrotron }{ }^{16} \\
\text { Ultrasound }{ }^{26}\end{array}$ & $\begin{array}{l}97 \\
92\end{array}$ & $\begin{array}{l}0.33 \\
0.352\end{array}$ \\
\hline $\mathrm{Mg}_{60} \mathrm{Cu}_{30} \mathrm{Y}_{10}$ & $\begin{array}{c}\text { Compression, synchrotron }{ }^{12} \\
\text { Ultrasound }^{22}\end{array}$ & $\begin{array}{l}64.1 \\
51.5\end{array}$ & $\begin{array}{c}0.373 \\
\mathrm{~N} / \mathrm{A}\end{array}$ \\
\hline $\mathrm{Zr}_{57} \mathrm{Ti}_{5} \mathrm{Cu}_{20} \mathrm{Ni}_{8} \mathrm{Al}_{10}$ & $\begin{array}{l}\text { Compression, synchrotron }{ }^{18} \\
\text { Macroscopic compression }^{27}\end{array}$ & $\begin{array}{l}87 \\
82\end{array}$ & $\begin{array}{c}0.34 \\
0.362\end{array}$ \\
\hline $\mathrm{Cu}_{47.5} \mathrm{Zr}_{47.5} \mathrm{Al}_{5}$ & $\begin{array}{c}\text { Compression, synchrotron }{ }^{17} \\
\text { Ultrasound }^{17}\end{array}$ & $\begin{array}{l}99.2 \\
90.1\end{array}$ & $\begin{array}{c}0.34 \\
0.365\end{array}$ \\
\hline $\mathrm{Zr}_{55} \mathrm{Cu}_{20} \mathrm{Ni}_{10} \mathrm{Al}_{10} \mathrm{Ti}_{5}$ & $\begin{array}{c}\text { Compression, synchrotron }{ }^{17} \\
\text { Ultrasound }{ }^{17}\end{array}$ & $\begin{array}{l}91.1 \\
85.5\end{array}$ & $\begin{array}{l}0.38 \\
0.378\end{array}$ \\
\hline
\end{tabular}

fact $^{13}$ we considered that here one can deal with two types of glasses: intrinsically brittle and plastic deformable in compression. Judging from the trend of the values of the elastic constants summarized in Table I, as measured by ultrasonic methods and as calculated from the strain tensor measured by diffraction experiments, it is very clear that in the case of "nondeformable" or intrinsically brittle BMGs, the bulk elastic constants as derived from ultrasound measurements and the ones obtained from the strain tensor analysis are almost similar, indicating a similar elastic behavior of each atomic shell. In the case of plastically deformable BMGs each atomic shell has a different stiffness, as revealed from the large differences of the elastic constants from ultrasonic and tensor analysis. Most likely, such local fluctuations of the elastic properties in the glassy structure can rather easily induce local shear transformation and, thus, the BMG exhibits macroscopic plasticity. ${ }^{13}$ However, in a glassy material a range of local atomic environments of the atoms exists. As a consequence of the disorder, fluctuation of inter-atomic distances may occur, which leads to variations of the atomic-level stress. ${ }^{16}$ The analysis of the first neighborhood, done by Mattern et al. ${ }^{16}$ for their CuZr binary glasses, confirms the anelastic changes of the short-range order under tensile stress well below the yield strength. The response of the nearest neighborhood upon loading leads toward the directional changes in the chemical shortrange order. All together may explain the differences in mechanical behavior observed at the microscopic level (x-ray diffraction) when compared with the averaged macroscopical behavior.

\section{CONCLUSIONS}

In-situ x-ray synchrotron diffraction enables the atomic level elastic strain of metallic glasses under uniaxial tensile or compressive stress to be characterized. The elastic moduli can be estimated not only considering the shift of the first maximum of the scattering curve in reciprocal space but also from the shift of the larger inter-atomic distances in the PDF in real space. The analysis of the short-range order of several metallic glasses vs. stress confirms the structural changes in the elastic regime. The anelastic deformations are accompanied by bond reorientation leading to direction dependent changes in chemical short-range order. The elastic constants calculated from the strain tensor are different from those measured by macroscopic techniques. These differences are supposed to arise because the ultrasound techniques average the elastic constants of different atomic shells and measure the bulk properties of the material, while the diffraction measurements may detect differences in stiffness of the first, second, third, and consecutive atomic shells.

\section{ACKNOWLEDGEMENTS}

The authors thank N. Mattern and S. Pauly for stimulating discussions. We thank also the beamline staff from BW5, Hasylab Hamburg, Germany and from IDI1, ESRF Grenoble, France, for their help during diffraction measurements. The authors are grateful for the financial support provided by the European Union within the framework of the Research Training Network on "Ductile BMG composites" (MRTN-CT-2003504692). The Alexander von Humboldt Foundation is acknowledged for financial support of $G$. Wang.

\section{References}

1.W.L. Johnson, MRS Bull., 24 (1999), p. 42.

2. A. Inoue, Acta Mater., 48 (2000), p. 279.

3. H. Chen et al., Nature, 367 (1994), p. 541.

4. S. Venkataraman et al., Scripta Mat., 54 (2006), p. 835.

5. M. Stoica et al., Intermetallics, 13 (2005), p. 764.

6. U. Kühn et al., Appl. Phys. Lett., 77 (2000), p. 3176.

7. J. Eckert, Mat. Sci. Eng. A, 226 (1997), p. 364

8. A.L. Greer, Science, 267 (1995), p. 1947.

9. L.Q. Xing et al., Phys. Rev. B, 64 (2001), 180201.

10.. J.J. Lewandowski and A.L. Greer, Nat. Mater., 5 (2006), p. 15

11. D.M. Dimiduk et al., Science, 312 (2006), p. 1188. 12. H.F. Poulsen et al., Nat. Mater., 4 (2005), p. 33

13. M. Stoica et al., J. Appl. Phys., 104 (2008) 013522.

14. J. Bednarčik and H. Franz, J. Phys.: Conf. Series, 144 (2009), 012058

15. X.D. Wang et al., Appl. Phys. Lett., 91 (2007), 081913.

16. N. Mattern et al., Acta Mat., 57 (2009), p. 4133. 17. J. Das et al., Phys. Rev. B, 76 (2007), 092203.

18. T.C. Hufnagel, R.T, Ott, and J. Almer, Phys. Rev. B, 73 (2006), 064204.

19. A.P. Hammersley et al., High Press. Res., 14 (1996), p. 235.

20. W. Dmowski and T. Egami, J. Mater. Res., 22 (2007), p. 412.

21. Y.H. Liu et al., Science, 315 (2007), p. 1385.

22. A. Castellero et al., J. Alloys and Comp., 434-435 (2007), p. 48.

23. J. Das et al., Phys. Rev. Lett., 94 (2005), 205501

24. K. Hajlaoui et al., J. Non-Cryst. Solids, 353 (2007) p. 327.

25. ASM Handbook: Volume 1.2 Metals Handbook (Materials Park, OH: ASM International, 1992).

26. G. Duan et al., Scripta Mater., 58 (2008), p. 159.

27. J.J. Lewandowski, W.H. Wang, and A.L. Greer, Philos. Mag. Lett., 85 (2005), p. 77.

Mihai Stoica and Gang Wang, researchers, and Jürgen Eckert, director, are with the Institute for Complex Materials, Helmholtzstr. 20, Dresden, 01069, Germany; Jayanta Das, senior research fellow, is with IIT Kharagpur, Department of Metallurgical and Materials Engineering, Kharagpur, India; Jozef Bednarčik, scientist, is with DESY, Hamburg, Germany; Gavin Vaughan, scientist, is with ESRF, Grenoble, France; and Wei Hua Wang, professor, is with the Institute of Physics, Chinese Academy of Sciences, Beijing, China. Dr. Stoica can be reached at m.stoica@ifw-dresden.de. 